\title{
Strain-Sensing Characteristics of Multi-Walled Carbon Nanotube Sheet
}

\author{
Daewoong Jung and Gil S. Lee
}

\begin{abstract}
In this paper, the properties of strain sensors made of spin-capable multi-walled carbon nanotubes (MWCNTs) were characterized and their sensing mechanisms analyzed. The key contribution of this paper is a new fabrication technique that introduces a simpler transfer method compared to spin-coating or dispersion CNT. Resistance of the MWCNT sheet strain sensor increased linearly with higher strain. To investigate the effect of CNT concentration on sensitivity, two strain sensors with different layer numbers of MWCNT sheets (one and three layers) were fabricated. According to the results, the sensor with a three-layer sheet showed higher sensitivity than that with one layer. In addition, experiments were conducted to examine the effects of environmental factors, temperature, and gas on sensor sensitivity. An increase in temperature resulted in a reduction in sensor sensitivity. It was also observed that ambient gas influenced the properties of the MWCNT sheet due to charge transfer. Experimental results showed that there was a linear change in resistance in response to strain, and the resistance of the sensor fully recovered to its unstressed state and exhibited stable electromechanical properties.
\end{abstract}

Keywords : Multi-walled carbon nanotube sheet, Strain sensor, Recovery response

\section{INTRODUCTION}

Strain sensors are important for weighing measurements, structural damage detection, and biomechanical applications [1]. The manufacture of a high-performance strain sensor is rather complex, as they must meet many requirements such as high sensitivity, linear response, fast response time, physical stability, wide operating range of strain, and low cost. To date, metal foil strain sensors have been widely used for measuring force and loading $[2,3]$. However, although metal-type sensors have many advantages, including low-cost, stability, and accuracy, they have several disadvantages such as limited monitoring locations, fixed directions, and low gauge factors. Moreover, fabrication of a metal strain sensor is difficult since they must be employed in a host composite.

Materials that have been studied for use as strain sensors include metal, optical fiber, metal oxide, and composites,

Department of Electrical Engineering, University of Texas at Dallas, 800 West Campbell Road, Richardson, TX 75080-3021, USA

${ }^{+}$Corresponding author: gslee@utdallas.edu

(Received: Aug. 1, 2013, Revised : Sep. 6, 2013, Accepted: Sep. 9, 2013)

This is an Open Access article distributed under the terms of the Creative Commons Attribution Non-Commercial License(http://creativecommons.org/licenses/bync/3.0)which permits unrestricted non-commercial use, distribution, and reproduction in any medium, provided the original work is properly cited. all of which have their own strong and weak points. Over the past two decades, nanostructure carbonaceous materials such as one-dimensional carbon nanotubes (CNTs) and graphene have shown great potential in strain sensor applications $[1,4,5]$. Compared to metal-like materials, nano-carbonaceous materials offer many advantages, such as higher mechanical stiffness, larger surface to volume ratio, and improved stability. Especially, the use of CNTs in sensing layers has been extensively studied, and many reviews have been reported on the development of CNTbased sensors [6, 7]. In recent years, CNTs have attracted interest in strain-sensing applications due to their high sensitivity and small size compared to conventional metalbased devices. Several groups have demonstrated the excellent piezoresistive properties of individual CNTs [4, 8]. The suitability of CNTs as strain sensors has been demonstrated both experimentally and theoretically. These studies have generally involved measuring the conductivity and/or resistance of individual CNTs under strain using an AFM tip or combined with micro-electro-mechanicalsystem (MEMS). In addition, CNT strain sensors are based on individual strands, assemblies, or dispersed solutions of single or multiwall tubes that may be functionalized by the embedded in suitable polymer or by addition of metal oxide nanoparticles $[9,10]$. Although individual CNTs 
have superior properties for sensor applications, their relatively poor process-ability and difficulty with regards to integration into bulk structures have hindered the mass production of practical strain sensors. Furthermore, to be used as a sensing layer, CNTs should be transferred from an initial substrate to another substrate (polymer or metal strain gage) using a complex transfer method, such as dispersion [1] or spraying [4]. Uneven dispersion and spraying of CNTs due to van der Waals forces can degrade sensor performance. These fabrication processes are complicated, time-consuming, and are thus not suitable for widespread use. Moreover, strain sensitivity is strongly dependent on the aspect ratio, density of the CNTs, as well as the properties of the polymers $[11,12]$. Therefore, simple, easy, low cost, highly productive, and reproducible fabrication techniques should be introduced for the successful fabrication of CNT-based sensors.

On the other hand, our group has previously reported MWCNT sheets fabricated from spin-capable MWCNT forests along with their applications [13-15]. MWCNT sheets have garnered much attention since the discovery that continuous sheets can be pulled from spin-capable MWCNT forests by van der Waals forces between MWCNTs. These MWCNT sheets consist of countless individual MWCNTs (inter-connections between MWCNTs) and show good adhesion to any substrate. These properties inspired us to apply MWCNT sheets to strain sensor applications. In this paper, a MWCNT sheetbased strain sensor is introduced along with a simple, easy transfer process that does not require manipulation of individual and/or bundles of CNTs or a complex, expensive fabrication process such as photolithography.

\section{EXPERIMENTAL}

\subsection{Materials and Sample Preparation}

An iron film was deposited onto $330 \mu \mathrm{m}$ thick p-type silicon wafers using electron beam (e-beam) evaporation. The thickness of the Fe films varied over a range of 5 to 6 $\mathrm{nm}$ and was monitored by a quartz-crystal sensor fixed inside the e-beam evaporation chamber. Catalyst annealing and CNT growth were performed in a vertical cylinder atmospheric-pressure chamber built by the authors. Flows of $\mathrm{He}(700 \mathrm{sccm}), \mathrm{C}_{2} \mathrm{H}_{2}(100 \mathrm{sccm})$, and $\mathrm{H}_{2}$ (100 sccm) were maintained using electronic mass flow controllers
(MFCs). After purging the tube with $\mathrm{He}$ for $10 \mathrm{~min}$, the chamber was ramped up to $780^{\circ} \mathrm{C}$ over $15 \mathrm{~min}$ (ramping rate: $50^{\circ} \mathrm{C} / \mathrm{min}$ ), after which growth of CNTs was carried out at $780^{\circ} \mathrm{C}$ by adding acetylene gas to the flow for $5 \mathrm{~min}$. Afterwards, the $\mathrm{C}_{2} \mathrm{H}_{2}$ and $\mathrm{H}_{2}$ gas flows were turned off and the sample rapidly cooled. As shown in Fig. 1, the MWCNT sheets were directly pulled from a super-aligned MWCNT forest onto PDMS. This provides a very simple and easy method to fabricate the strain sensor. In order to enhance the adhesion between the sheet and substrate, ethanol was dropped onto the entire surface of the MWCNT sheet on the substrate and dried at ambient temperature for 1 hour.

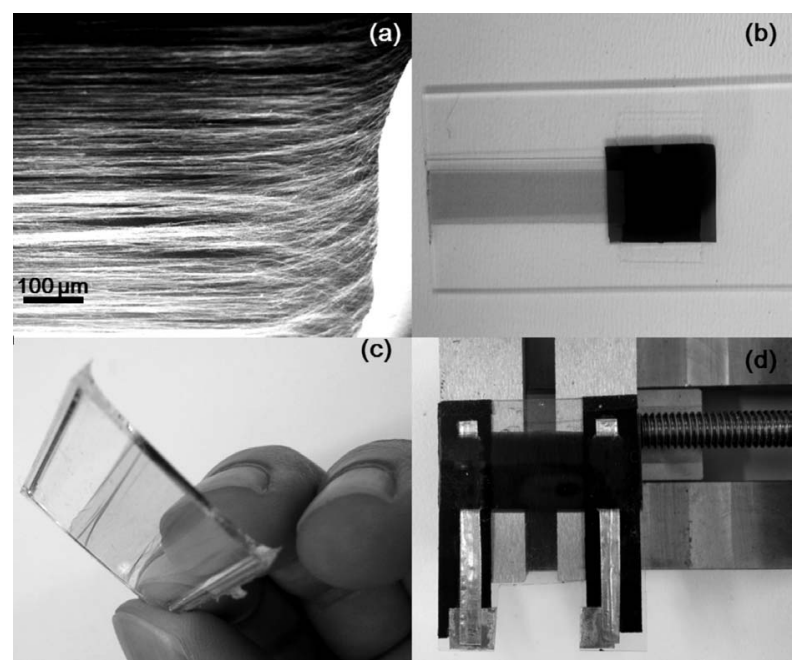

Fig. 1. SEM image and photographs of (a) Spin-capable CNTs showing good alignment of MWCNTs, (b) CNT sheet pulling from a CNT forest, sheet transfer from the $\mathrm{Si}$ substrate to glass, (c) PDMS substrate, and (d) fabricated strain sensor.

\section{RESULTS AND DISCUSSIONS}

\subsection{Strain-sensing response}

To evaluate strain-sensing characteristics, the sensor was fastened firmly with two metal grips. One grip was fixed in order to ensure that the sample was in a standard position; the other was used to apply strain. The distance used to apply the strain was controlled manually. The sensitivity of the sensor was determined using the following equation:

$$
\text { Sensitivity }(\%)=\left(\Delta \mathrm{R} / \mathrm{R}_{0}\right) /\left(\Delta \mathrm{L} / \mathrm{L}_{0}\right)
$$


where $\Delta R / R_{0}$ is the relative change in resistance of the sensor before and after strain $\left(\Delta \mathrm{L} / \mathrm{L}_{0}\right)$. The sensitivity of the MWCNT sheet strain sensor was observed by measuring the variation in resistance as a function of changing strain. Figure 2 shows the typical strain-sensing response of the sensor to various strain levels. The resistance of the strain sensor proportionally increased with increasing strain level. The strain-sensing mechanism of the sensor could be understood as follows [16]. In principle, the piezo-resistivity of a sensor can be mainly attributed to the variation of CNT distribution under applied strain. The total resistance $\left(\mathrm{R}_{\text {total }}\right)$ of the MWCNT sheet is illustrated in Fig. 3. $\mathrm{R}_{\text {initial }}$ is the resistance along the entire length of the MWCNT sheet before the strain, and $\mathrm{R}_{\text {strain }}$ is the change in contact resistance induced by the applied strain between individual MWCNTs. When strain was subjected to the sensor, some of the MWCNTs might have disconnected, thereby increasing $\mathrm{R}_{\text {total }}$. As greater strain was applied, more CNTs became disconnected, thereby resulting in a larger change in resistance, as shown in Fig. 2. Therefore, changes in the contact area within the MWCNT sheet under strain led to significant changes in electrical resistance, resulting in increased sensitivity of the strain sensor.

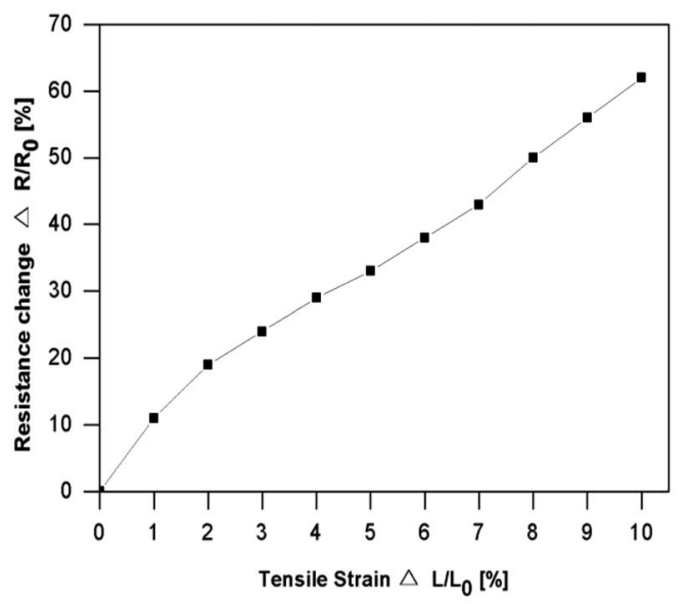

Fig. 2. Change in resistance in a MWCNT sheet as a function of tensile strain.

Besides the inter-connection model, other mechanisms of strain-sensing have been proposed. Paulson et al [17] were successful in measuring the resistance of a CNT under in situ strain, and they observed that the resistance increased by a few orders of magnitude when the nanotube was subjected to strain. It has been proven mathematically by several groups $[18,19]$ that the electronic energy band gap of CNTs increases with uniaxial and torsional strain. As a MWCNT sheet contains many individual CNTs, the change in resistance of a sensor is a collective representation of all individual CNTs in the sheet. In other words, the band gap energy of individual CNTs in the sheet increases under applied strain, which leads to increasing total resistance of the sheet. Therefore, our experimental results are in line with the theoretical conclusions given by Paulson's groups.

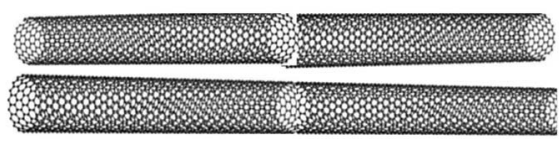

(a) $R_{\text {total }}=R_{\text {initia }}$

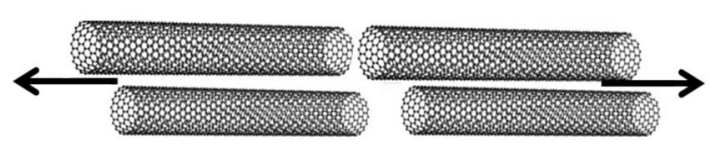

(b) $\mathrm{R}_{\text {total }}=\mathrm{R}_{\text {initial }}+\mathrm{R}_{\text {strain }}$

Fig. 3. Visualization of the operating principle; (a) before and (b) after strain.

\subsection{Effect of contact area of MWCNTs on strain- sensing response}

In order to investigate the correlation between concentration of CNTs and sensor sensitivity, the two types of samples processed with different numbers of sheet layers were prepared on PDMS substrates by overlaying the layers one and three times. Figure 4 shows the sensitivity of the sensor as function of strain with respect to the number of layers. As expected, the concentration of individual CNTs in the sheet influenced the sensitivity of the strain sensor. It was found that the sensitivity of the sensor increased with increasing number of MWCNT sheet films. For example, the sensitivity of the sensor with a three-layer sheet was nearly $20 \%$ greater than that of the sensor with a one-layer sheet. As mentioned, a MWCNT sheet is formed by hundreds of thousands of individual CNTs, and the density of CNTs in the sheet is sustained by van der Waals forces. Therefore, the contact area rapidly increases as the number of layers increases. It is well known that the number of contact points between individual CNTs (inter-tube contacts) is a critical factor for determining the performance of CNT-based sensors [7]. 
Therefore, the above enhancement in sensitivity was due to the fact that an increased number of contact points between MWCNTs improves sensor sensitivity. Overall, although the sensitivities of the both sensors were not perfectly linear with tensile strain, sensor with one-layer shows better linearity than that with three-layers.

\subsection{Reproducibility and Stability}

Major problems to be solved relating to CNT-based sensors include reproducibility, which refers to multiple resistance-strain behaviors under cyclic operating conditions, and stability, which refers to stable resistance over a long time. In the past, the resistance of CNT sensors has varied in response to a zero load in repeatable tests [20, 21], which is a serious problem with regards to practical applications. This could be due to the permanent deformation of individual CNTs resulting from their lower density. CNT strain sensors often show resistance hysteresis under cyclic operating conditions, resulting in irreversible degradation of CNT-CNT interfaces. Moreover, the resistance of a sensor is unstable, even without an applied mechanical load [28, 29].

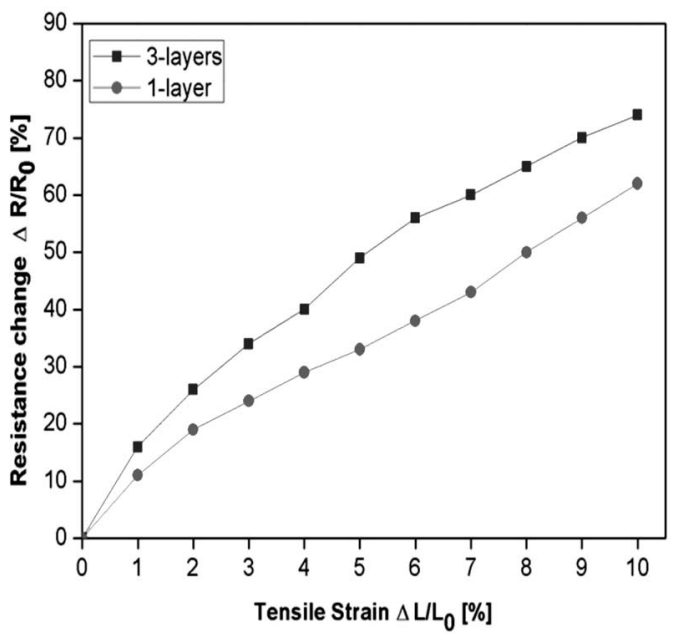

Fig. 4. Output characteristics of the MWCNT sheet strain sensor as a function of the number of layers.

To investigate the reproducibility of the sensor, multiple cycles were carried out using cyclic stretching and relaxation. As shown in Fig. 5 (a), the sensor response was reproducible with negligible hysteresis and recovered to its initial value. Regarding stability, the performance of the sensor remained stable over a long time period, as shown Fig. 5 (b). As mentioned previously, CNT sheets contain thousands of short, individual CNTs that are several hundred micrometers long (330 $\mu \mathrm{m}$ in this study). A densely packed, large contact area along the entire CNT length combined with large van der Waals forces between the CNTs ensured the structural stability of the sheet during stretching and relaxation. The stability of the sensor can be attributed to the steady and large contact area between the CNTs made possible through strong van der Waals forces. Moreover, the large CNT contact area prevented chargeinduced contact damage. Sensors fabricated by conventional CNT dispersal or spin-coating have fewer contact points and large current concentrations, which can result in deterioration due to localized heating [22]. In contrast, our MWCNT sheets had a large contact area, leading to less localized heating and improved long-term resistance stability.

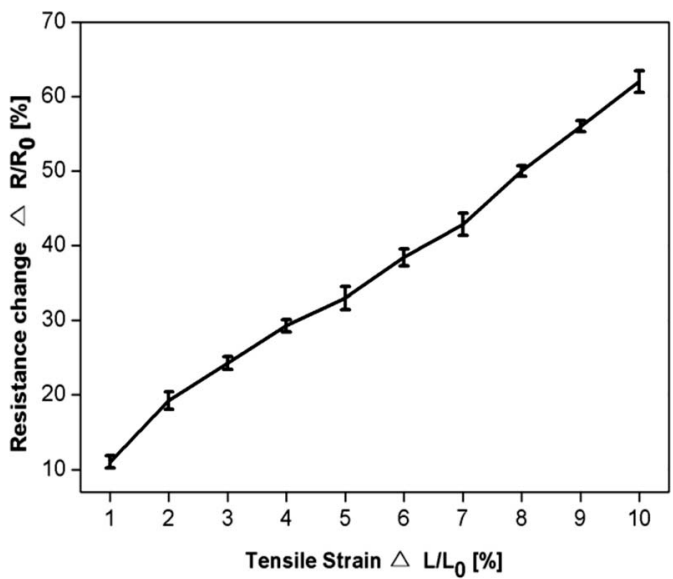

(a)

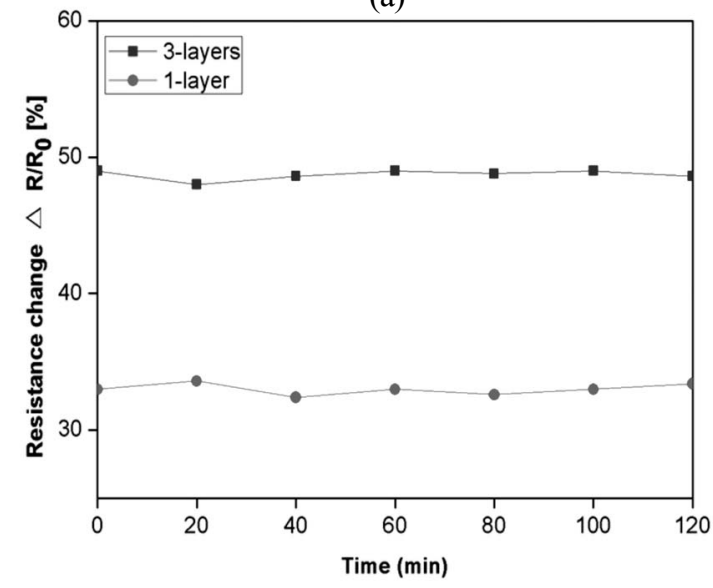

(b)

Fig. 5. (a) Hysteresis measurement of the MWCNT sheet strain sensor and (b) long-term stability results.

\subsection{Effects of environmental factors on strain-sensing response}


Further experiments were carried out to investigate environmental effects such as temperature and gas. First, temperature plays a vital role in determining the performance of a sensor. Especially, CNT-based sensors are very sensitive to environmental temperature due to their intrinsic nature, as expansion/shrinkage of the substrate caused by variation in temperature will be detected as strain by the sensor [23]. The effect of temperature on the sensitivity of the sensor was measured from room temperature $\left(22^{\circ} \mathrm{C}\right)$ to $50{ }^{\circ} \mathrm{C}$, and the output result is shown in Fig. 6. The plot indicates that the sensitivity of the sensor to strain decreased as the temperature increased. This can be explained by the fact that a higher temperature can reduce electronic energy band-gap of CNTs. In other words, increased band-gap induced by strain was compensated for at high temperature, which reduced the sensitivity of the sensor. In strain-sensing, individual CNTs should be physically connected in order to form electronconducting ways or at least be close enough to allow electron transfer (tunneling effect) before the strain is applied [32]. Further, electrons can jump long distances between CNT-CNT junctions due to the high kinetic energy of electrons at high temperature, which causes smaller changes in resistance under a given strain.

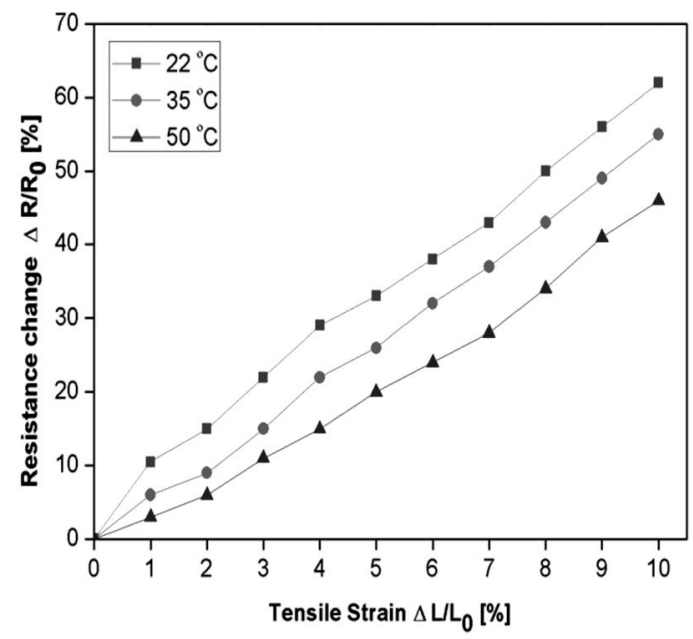

Fig. 6. Output characteristics of the MWCNT sheet strain sensor as a function of the number of layers.

Second, the conductivity of CNTs can be altered upon exposure to gases due to charge transfer between the CNTs and gas molecules [19]. For example, when the sensor was exposed to an exhaled breath, the resistivity changed by $0.8 \%$, (not shown here), thereby affecting the performance of the strain sensor. Therefore, packaging must be considered for reducing environmental effects as well as to prevent damage from corrosion.

\section{CONCLUSIONS}

Piezo-resistive properties of a MWCNT sheet-based strain sensor were investigated and characterized under tensile strain up to $10.0 \%$. Resistance of the MWCNT sheet sensor increased when the level of applied strain increased. The strain characteristics of the sensor showed a linear response, excellent reproducibility, and small hysteresis, and its strain sensitivity was stable over a long time period. A novel transfer method was introduced to fabricate a CNT-based sensor without any complex fabrication process such as photolithography. Our fabricated MWCNT sheet can be extended to other CNTbased applications (gas, humidity, pH sensor, and electrodes) as well as to the construction of a flexible sensing material.

\section{ACKNOWLEDGMENT}

The authors thank DMS for donating the CVD.

\section{REFERENCES}

[1] Y. Wang, A. X. Wang, Y. Wang, M. K. Chyu, and Q. M. Wang, "Fabrication and characterization of carbon nanotube-polyimide composite based high temperature flexible thin film piezoresistive strain sensor", Sens. Actuators A-Phys., Vol. 199, pp. 265-271, 2013.

[2] A. Karaus and H. Paul, "Load cells with small nominal load based on strain gauges using thin-film techniques", Measurement, Vol. 10, pp. 133-139, 1992.

[3] C. K. Demetropoulos, C. R. Morgan, D. K. Sengupta, and H. N. Herkowitz, "Development of a 4-axis load cell used for lumbar inter body load measurements", Med. Eng. Phys., Vol. 31, pp. 846-851, 2009.

[4] Z. L. Li, P. Dharap, S. Nagarajaiah, E. V. Barrera, and J. D. Kim, "Carbon nanotube film sensors", $A d v$. Mater., Vol. 16, pp. 640-643, 2004.

[5] S. H. Bae, Y. Lee, B. K. Sharma, H. J. Lee, J. H. Kim, and J. H. Ahn, "Graphene-based transparent strain sensor", Carbon, Vol. 51, pp. 236-242, 2013. 
[6] D. Jung, K. H. Lee, D. Kim, D. Burk, L. J. Overzet, and G. S. Lee, "Highly conductive flexible multi-walled carbon nanotube sheet films for transparent touch screen”, Jpn. J. Appl. Phys., Vol. 52, p. 03BC03, 2013.

[7] K. S. Karimov, M. T. S. Chani, F. A. Khalid, and A. Khan, "Strain sensors based on carbon nanotubescuprous oxide composite", Physica E, Vol. 44, pp. 778781, 2012.

[8] X. Li, C. Levy, and L Elaadil, "Multiwalled carbon nanotube film for strain sensing", Nanotechnology, Vol. 19, p. 045501, 2008.

[9] K. J. Loh, J. Kim, J. P. Lynch, N. W. S. Kam, and N. A. Kotov, "Multifunctional layer-by-layer carbon nanotube-polyelectrolyte thin films for strain and corrosion sensing", Smart Mater. Struct., Vol. 16, pp. 429-438, 2007.

[10] I. Kang, M. J. Schulz, J. H. Kim, V. Shanov, and D. Shi, "A carbon nanotube strain sensor for structural health monitoring", Smart Mater. Struct., Vol. 15, pp. 737-748, 2006.

[11] K. J. Loh, J. P. Lynch, and N. A. Kotov, "Conformable single-walled carbon nanotube thin film strain sensors for structural monitoring", Proc. of the 5th Int. Workshop on Struct. Health Monit., pp. 18. 2005.

[12] S. M. Vemuru, R. Wahi, S. Nagarajaiah, and P. M. Ajayan, "Strain sensing using a multi walled carbon nanotube film", J. Strain Anal. Eng. Des., Vol. 44, pp. 555-562, 2009.

[13] J. H. Kim, K. H. Lee, D. Burk, L. J. Overzet, and G. S. Lee, "Tuning of Fe catalysts for growth of spincapable carbon nanotubes", Carbon, Vol. 48, pp. 538$547,2010$.

[14] D. Jung, D. Kim, K. H. Lee, L. J. Overzet, and G. S. Lee, "Transparent film heaters using multi-walled carbon nanotube sheets", Sens. Actuators A-Phys., Vol. 199, pp. 176-180, 2013.
[15] D. Jung, Y. Yoon, and G. S. Lee, "Hydrogen sensing characteristics of carbon-nanotube sheet decorated with manganese oxides", Chem. Phys. Lett., Vol. 577, pp. 96-101, 2013.

[16] H. Zhao, Y. Zhang, P. D Bradford, Q. Zhou, Q. Jia, F.-G. Yuan, and Y. Zhu, "Carbon nanotube yarn strain sensors", Nanotechnology, Vol. 21, p. 305502, 2010.

[17] S. Paulson, M. R. Falvo, N. Snider, A. Helser, T. Hudson, A. Seeger, R. M. Taylor, and S. Washburn, "In situ resistance measurements of strained carbon nanotubes”, Appl. Phys. Lett., Vol. 75, pp. 2936-2936, 1999.

[18] S. V. Anand and D. R. Mahapatra, "Quasi-static and dynamic strain sensing using carbon nanotube/epoxy nanocomposite thin film", Smart Mater. Struct., Vol. 18, p. 045013, 2009.

[19] H. Jiang, Y. Zhang, G. Yu, and J. Dong, "The effect of uniaxial and torsional deformations on quantum interference of carbon nanotubes", Phys. Lett. A, Vol. 351, pp. 308-313, 2006.

[20] K. J. Loh, J. Kim, J. P. Lynch, N. W. S. Kam, and N. A. Kotov, "Multifunctional layer-by-layer carbon nanotube-polyelectrolyte thin films for strain and corrosion sensing", Smart Mater. Struct., Vol. 16, pp. 429-438, 2007.

[21] E. T. Thostenson and T. W. Chou, "Real-time in situ sensing of damage evolution in advanced fiber composites using carbon nanotube networks", Nanotechnology, Vol. 19, pp. 215713-215419, 2008.

[22] F. Zheng, Z. Zhou, X. Yang, Y. Tang, and Y. Wu, "Sorting single-walled carbon nanotubes by strainbased electrical burn-off", Carbon, Vol. 48, No. 8, pp. 2169-2174, 2010.

[23] C. L. Cao, C. G. Hu, Y. F. Xiong, X. Y. Han, Y. Xi, and J. Miao, "Temperature dependent piezoresistive effect of multi-walled carbon nanotube films", Diam. Relat. Relat. Mater., Vol. 16, pp. 388-392, 2007. 